\title{
L'Ovide moralisé et la tradition encyclopédique médiévale
}

Une approche générique comparative

\section{Bernard Ribémont}

\section{(2) OpenEdition}

\section{Journals}

Édition électronique

URL : https://journals.openedition.org/crm/907

DOI : $10.4000 / \mathrm{crm} .907$

ISSN : 1955-2424

\section{Éditeur}

Honoré Champion

Édition imprimée

Date de publication : 10 décembre 2002

ISSN : 1272-9752

\section{Référence électronique}

Bernard Ribémont, «L'Ovide moralisé et la tradition encyclopédique médiévale », Cahiers de recherches médiévales [En ligne], 9 | 2002, mis en ligne le 04 septembre 2007, consulté le 15 décembre 2022 URL : http://journals.openedition.org/crm/907 ; DOI : https://doi.org/10.4000/crm.907

Ce document a été généré automatiquement le 15 décembre 2022.

Tous droits réservés 


\section{L'Ovide moralisé et la tradition encyclopédique médiévale}

Une approche générique comparative

\section{Bernard Ribémont}

1 L'Ovide moralisé est d'une ampleur tout encyclopédique : les cinq volumes de la version en vers dans l'édition De Boer ${ }^{1}$ ont de quoi impressionner le lecteur désireux de connaître la réception d'Ovide à la fin du Moyen Âge. Ce texte tient de la somme ${ }^{2}$, de la glose, de l'encyclopédie moralisée, de la translation - au sens médiéval - d'un texte latin et, pour le moins, il s'organise en lieu de mémoire ${ }^{3}$. À la suite de cette brève énumération, on peut mesurer que ce texte est difficile à classer d'un point de vue générique : on peut fort bien le considérer comme une compilation de mythes ovidiens ; ces mythes étant moralisés, on peut rapprocher l'ouvrage du Bestiaire - les techniques d'écriture pouvant paraître proches -, de l'encyclopédie moralisée ${ }^{4}$, ces deux genres d'ailleurs étant connexes ${ }^{5}$.

2 Je voudrais dans cet article m'intéresser à la question du genre de l'Ovide moralisé. On sait que cette question de l'établissement des genres est particulièrement complexe pour ce qui concerne la période médiévale. Pour Paul Zumthor, la recherche des genres est source de «malentendus » et repose surtout sur une conception post-médiévale rapporté «à un fonds doctrinal aristotélicien $»^{6}$. Mais Ernst Robert Jauss a cependant insisté, tout en remettant en cause l'acception classique de genre, sur l'importance d'une analyse générique, en particulier du point de vue de la réception, et il aboutit alors à la considération d'une "famille historique» conditionnée par un horizon d'attente ${ }^{7}$. Il est a priori délicat de vouloir situer l'analyse générique de l'Ovide moralisé dans une perspective authentiquement diachronique ou, selon ce que propose également Jauss, dans l'évolution de coupes synchroniques. On ne peut en effet compter que sur la version en vers et sur sa mise en prose, fortement résumée, du XV siècle ${ }^{8}$.

3 Après Tynjanov, Jauss a introduit la notion de «dominante »" qui va orienter ici ma recherche. Plutôt que de parler péjorativement de «mélange des genres », l'on partira du constat qu'il peut exister dans une œuvre ou un ensemble d'œuvres un noyau 
générique cristallisateur, autour duquel plusieurs genres ou sous-genres peuvent se greffer de manière concomitante ou constitutive (d'un nouveau genre). Jauss propose l'exemple du Roman de la Rose de Jean de Meun, dont la dominante est l'« encyclopédie laïque $»^{10}$.

Mon propos ne sera donc pas de définir le genre à proprement parler de l'Ovide moralisé, ce qui serait sans doute une entreprise vouée à l'échec: plus exactement, le résultat de l'enquête pourrait bien être que cette œuvre appartient au genre de ...l'ovide moralisé ! Je voudrais plutôt considérer une dominante, dans un sens un peu différent de celui de Jauss, car il s'agira d'une dominante externe à l'œuvre elle-même. Cette dominante sera le genre encyclopédique ${ }^{11}$. Pour justifier cette enquête et sa méthode, j'avancerai trois arguments principaux :

5 1) l'Ovide moralisé, comme les textes encyclopédiques, a servi de "réservoir de données " aux auteurs familiers de la compilation et de l'écriture poétique à visée didactique, comme Guillaume de Machaut, Eustache Deschamps ou Christine de Pizan ${ }^{12}$;

6 2) les principes de moralisation mis en œuvre dans l'Ovide moralisé reposent sur les mêmes principes que ceux des Bestiaires ou des Lapidaires, textes renvoyant à la tradition encyclopédique ;

7 3) l'Ovide moralisé se présente comme une somme «scientifico-mythologique » dans laquelle - au niveau des moralisations - fonctionne un processus de transposition de savoir proche de la transposition encyclopédique ${ }^{13}$.

8 La méthode employée sera donc comparative, dans le sens suivant : en partant du fait que l'ovide moralisé possède un certain nombre de fonctionnalités, de façon interne comme dans sa réception, qui ressortissent au genre encyclopédique, je tenterai de définir quels liens de connexité ce texte entretient avec la tradition encyclopédique médiévale. L'intérêt de définir de tels liens réside, non dans une définition générique de l'Ovide moralisé - probablement illusoire on l'a dit-, mais dans la détermination de la place qu'un tel ouvrage peut occuper dans le champ littéraire de la fin du Moyen Âge.

Réception

9 Une étude détaillée et approfondie de la réception de l'Ovide moralisé reste à faire. Il apparaît cependant que son succès à la fin du Moyen Âge est une chose clairement attestée. Comme l'avait bien noté son éditeur, l'Ovide moralisé, version rimée, fut largement reçu comme un ouvrage où de nombreux auteurs, tels Machaut, Froissart, Deschamps ou Christine de Pizan puisèrent des exemples mythologiques venant illustrer leur propre texte. On sait, après le Roman de la Rose et, particulièrement après Machaut, combien grande fut la «mode» de l'insertion d'exemples mythologiques, voire même d'inventions mythologiques ${ }^{14}$ dans la tradition littéraire médiévale ${ }^{15}$. Dans ce contexte, l'Ovide moralisé occupe une place essentielle, véritable mémoire, dans tous les sens du terme, lieu privilégié où le mythe se dit, se raconte, et même s'interprète.

On relèvera ici que la moralisation du mythe n'est pas ce qui a retenu particulièrement l'attention des lecteurs-poètes. Si l'on trouve sous la plume de nos auteurs des exemples de mythologie ovidienne, il est bien rare que ceux-ci soient donnés glosés. L'importance accrue de la mythologie dans l'écriture médiévale est à mettre en relation, toujours à partir du XIVe siècle, avec un net renforcement des tendances didactiques des écrivains. Cet infléchissement aboutit, en particulier, à la multiplication de ce que j'ai appelé des «insertions savantes $»^{16}$. Les auteurs, dans un souci d'affirmer leur autorité, comme dans celui de mieux illustrer leur discours à l'aide 
d'exemples, s'alimentent à une tradition encyclopédique, d'ailleurs foisonnante, qui leur offre de multiples possibilités en ce domaine. Les textes encyclopédiques médiévaux répondent alors à un besoin essentiel, qui dépasse celui de lettrés en quête d'un savoir sur la nature: ils apparaissent en ce contexte comme de véritables «réservoirs", des lieux de mémoire, j'oserai même parler de database, pour des auteurs en quête d'images et d'exemples. Et l'on enregistre bien, chez des écrivains dont l'œuvre didactique est majeure, comme Christine de Pizan par exemple, une fréquentation régulière des ouvrages encyclopédiques ${ }^{17}$.

11 À ce niveau, un parallèle entre les textes encyclopédiques et notre Ovide moralisé paraît légitime, sans aller plus loin dans une analyse de contenu : en terme de réception, du texte encyclopédique est extrait, "pour les besoins de la cause", une information nécessairement parcellaire, à contenu lié aux choses de la nature; de l'Ovide moralisé sera tiré un ensemble, lui aussi parcellaire, de données mythologiques. Ce sont ces données que les poètes utilisent, se livrant effectivement à une extraction. Dans la mesure où, effectivement, la moralisation n'est pas retenue. Dans ce contexte de "réception littéraire», on peut s'interroger sur la fonction réelle de la moralisation. Sans doute serait-il quelque peu absurde de remettre en cause la bonne foi moralisatrice de l'auteur de l'Ovide moralisé. Cependant, on notera que, dans le prologue, l'auteur n'insiste pas particulièrement sur cette «mutacion» morale de la fable. Il entend donner celles (les «mutacions ») qui sont «bones et profitables» (v. 54), "pour plus plaire a ceulz qui l'orront». La moralisation prend alors, explicitement, deux fonctions: édificatrice et ornementale. Vue du côté de l'ornement, l'allégorie édifiante ne se ramène-t-elle pas alors à un jeu d'écriture ? Moraliser serait donc, aussi, montrer son habileté à un jeu déjà fort ancien, bien attesté.

12 À quel public l'auteur s'adresse-t-il ? On peut penser à des clercs en premier lieu. C'est d'ailleurs ce que l'auteur avoue lui-même, en s'adressant à «Tous les maistres, tous les rectors/Qui orront et liront ce dit» (XV, vv. 7474-75). Mais à des clercs d'un niveau moyen, même si la mention de recteurs peut faire penser à des docteurs de haut niveau; sinon, à quoi bon traduire, là où il suffirait de moraliser? Plus largement, cet auteur qui entend aussi «plaire» vise un public plus large qu'il veut ainsi éduquer en le distrayant. Dès lors, et la réception « littéraire » de l'œuvre le prouve, l'auteur de l'Ovide moralisé met en place un processus qui autorise une indépendance entre la fable et son allégorie. Mais une lecture discontinue de l'œuvre n'est pas explicitement encouragée pour autant. L'allégorie peut alors apparaître, dans une certaine réception, comme le soulignement de la fable, le tout formant une unité plus aisément mémorisable.

Par ailleurs, l'Ovide moralisé a été glosé, comme le révèlent les notes marginales des manuscrits. Ces notes de lecture fournissent de précieuses indications sur la manière dont le texte a pu être reçu ${ }^{18}$. Il ressort de l'analyse de ces marginalia que :1) la plupart des notes sont en latin; 2) les gloses marginales portent dans leur grande majorité sur la moralisation, ajoutant bien souvent des renvois aux Écritures saintes. Une longue glose en français, reposant sur l'image des flèches de Cupidon, peut d'ailleurs apparaître comme une véritable moralisation d'un passage du Roman de la Rose, selon des principes que l'on retrouve dans les Échecs moralisés d'Evrart de Conti. Les lecteurs ont donc eu le désir de compléter, de préciser certaines allégations de l'auteur de l'Ovide moralisé à propos d'interprétation chrétienne. 

dans le milieu des clercs de la fin du Moyen Âge. Le latin leur est plus familier que le vernaculaire et c'est la moralisation chrétienne qu'ils recherchent en premier lieu. L'étude (encore partielle) des notes marginales des manuscrits d'encyclopédies aboutit à des conclusions analogues ${ }^{19}$. On peut donc établir à ce niveau encore un parallèle entre les encyclopédies et l'Ovide moralisé, dans la mesure où ces deux catégories de texte, du point de vue de leur réception, répondent à des attentes similaires dans le milieu des clercs. L'Ovide moralisé, comme les encyclopédies moralisées, offre des unités de savoir à caractère double. Mais, cette fois-ci, la mémorisation obéit à un processus différent (théoriquement) de celui mis en œuvre pour la réception des poètes : c'est la fable qui sert de point de fixation de l'allégorie.

finir, considérons un dernier témoignage de la réception de l'Ovide moralisé du $\mathrm{XIV}^{\mathrm{e}}$ siècle : il s'agit de sa mise en prose $\mathrm{du} \mathrm{XV}^{\mathrm{e}}$ siècle. Rappelons qu'un auteur, qui se donne pour normand, met en prose l'Ovide moralisé, en 1466, pour René d'Anjou ${ }^{20}$. Il indique, dans un "prologue de celluy qui a converti de rime en prose françoise le livre d'Ovide appellé methamorphoses » qu'il entend exposer sa matière « soubz le plus brief langaige de prose » (p.43) qu'il lui a été possible de produire, «sans y laisser aucunes places pour faire hystoires ». En toute conscience donc, et, semble-t-il, sous les ordres $\mathrm{du}$ roi René ${ }^{21}$, l'auteur entend résumer sa source, tout en participant du topos encyclopédique de la brièvetée ${ }^{22}$. Comme le remarque C. de Boer (éd. cit., pp. 12-4), ce résumé, parfois drastique, vise essentiellement les allégories. De plus, si l'on considère les ajouts et modifications faits par le prosateur, ils ont pour la plupart, non des sources bibliques, mais des textes profanes, de Boccace en particulier. Enfin, les quelques notes marginales du manuscrit, très peu nombreuses, se partagent entre latin et français, sont brèves et n'ont jamais de caractère moralisant. Nous avons donc indéniablement affaire à une mise en prose qui se veut simplificatrice, axée essentiellement sur la mise en mémoire des fables ovidiennes. L'auteur obéit à la volonté de son mécène d'une véritable vulgarisation, au sens moderne de ce terme, de la mythologie ovidienne, démarche qui, là encore, nous rapproche de l'encyclopédisme et de la vulgarisation à la fin du Moyen Âge. Cette vulgarisation encyclopédique correspond à ce que nous appelons aujourd'hui une traduction. L'exemple de la translation du De proprietatibus rerum montre bien que la vulgarisation de Corbechon doit s'entendre comme un mélange des deux sens du terme, médiéval et moderne. Car la traduction depuis le latin implique aussi un résumé, une adaptation à un public plus large. Comme dans le passage du vers à la prose pour l'Ovide moralisée ${ }^{3}$.

On pourrait s'interroger sur le fait que l'Ovide moralisé en prose n'est pas une traduction des Métamorphoses, mais une adaptation du texte moralisé. D'autant plus que, comme le montre l'éditeur du texte, l'auteur du XV siècle a probablement utilisé le texte latin d'Ovide. On peut considérer que l'auteur au service du roi René est plus poète que clerc, et que le choix d'un hypotexte en vernaculaire lui facilite la tâche. Certes, mais la justification parait bien courte. Je crois qu'il faut davantage penser, encore une fois, en termes de réception. La vulgarisation, largement encouragée par les puissants après le développement des traductions qui s'opère durant le règne de Charles $V$, s'inscrit dans un «espace de savoir", conditionné - pour reprendre des termes chers à Pierre Bourdieu - par un "marché du savoir», en mutation. Si la vulgarisation (au sens médiéval) d'ouvrages scientifiques, encyclopédiques, au moins reçus comme tels, répond à une réelle demande d'un public cultivé, si elle est aussi motivée en amont par 
des considérations politiques, elle est partie prenante du mouvement culturel qui annonce et prépare l'humanisme. Mais, justement, dans le domaine de la vulgarisation. C'est-à-dire avec de nombreux décalages par rapport au monde du "savoir savant", d'ailleurs animé lui aussi de nombreuses tensions. Le texte vulgarisé participe de ce champ de tension, tout en restant ancré bien davantage dans une problématique de l'autorité, dans un conservatisme parfois frileux. L'Ymago mundi de Pierre d'Ailly est écrite en latin, au $\mathrm{XV}^{\mathrm{e}}$ siècle, et ne fournit guère plus d'informations que le Urtext d'Honorius, au XII siècle : latin savant, vulgarisation de très bas niveau: pour quel public ? ${ }^{24}$ La vulgarisation navigue donc, si j'ose dire, avec difficulté, au milieu de tendances fortement contradictoires. Un élan du français, avec aussi un goût de certains auteurs pour les latinismes ${ }^{25}$, un retour au «beau latin » (Nicolas de Clamanges, Laurent le Premierfait, etc.), un bilinguisme revendiqué (Gerson $\left.{ }^{26}\right)$, un déclin de la méthode allégorique, qui, toutefois, se maintient encore très largement. Cette dernière tension nous ramène encore au genre encyclopédique. L'encyclopédie isidorienne, puis le mouvement post-isidorien ${ }^{27}$ sont marqués par l'exposé d'un savoir véritablement positif. En cela, si ces encyclopédies répondent à un besoin, elles soulignent aussi un manque, comme le montrent les différentes moralisations, - songeons à l'œuvre de Pierre Bersuire qui moralise le De proprietatibus rerum - et les marginalia des manuscrits. Les œuvres à caractère encyclopédique sont donc reçues par des publics aux attentes différentes. Plus exactement, elles suscitent des lectures diverses, dont les tendances différentes, voire contradictoires, s'entremêlent, dans une durée qui voit se construire progressivement, dans les milieux savants et lettrés, un courant humaniste, lui aussi animé de mouvements contradictoires.

On peut donc penser que l'auteur de l'Ovide moralisé en prose choisit un milieu des choses. Il propose un recueil de fables ovidiennes, en français, mémoire mythologique condensée; il conserve une part de la moralisation, à cause de l'autorité du textesource, pour fournir aussi un lieu de mémoire de certaines allégories, par souci d'orthodoxie, par maintien d'une tradition qu'il ne se sent pas en devoir ou en mesure de ne pas suivre.

En conclusion de ces remarques comparatistes sur la réception de l'œuvre, l'ovide moralisé, comme les textes encyclopédiques, fonctionne comme réservoir d'informations pour deux démarches distinctes. D'une part, des écrivains, des poètes, soucieux d'autorité et de didactisme, vont chercher une information "positive", à partir de laquelle ils pourront pratiquer une insertion savante ou mythologique. D'autre part, des clercs lisent ces textes à la recherche d'exempla et/ou de codes pour une lecture et une exégèse des Écritures. Quant à sa réception, l'Ovide moralisé peut donc apparaître en quelque sorte comme la mémoire encyclopédique ovidienne de la fin du Moyen Âge, dans une tentative qui, en ces temps de pré-humanisme, demeure très médiévale, par le fait même de la moralisation.

Moralisation

Pour un lecteur moderne, les parties allégoriques d'un ouvrage didactique médiéval sont souvent d'un abord difficile, disons surtout étrange. Ledit lecteur est en effet en général déconcerté par ce qui lui apparaît comme incongru: dire que la panthère représente le Christ, ou Daphné la sainte Vierge peut en effet, pour le moins, être mis au rang d'une déconcertante naïveté. Le lecteur non averti conclura, une fois de plus, à l'obscurantisme; celui, plus médiéviste, qui refuse de tels raccourcis, pensera davantage à cet univers symbolique prêté à une époque où Dieu est partout. Cependant, 
point n'est besoin de grandes démonstrations pour prouver que la pensée du Moyen Âge a poussé très loin l'exercice de la raison et il me paraît bien léger de considérer que tous les auteurs de Bestiaires ou d'encyclopédies moralisées ont pu se contenter, sans une raison valable, de répéter des correspondances, dont beaucoup sont «tirées par les cheveux ». Un auteur comme Richard de Fournival, au demeurant fort savant homme, n'indique-t-il pas, en jouant sur la moralisation, qu'il peut s'agir aussi d'un jeu d'écriture, en l'occurrence d'un jeu poétique, casuistique et rhétorique, comme il le montre dans son Bestiaire d'amour, qui obéit à une construction très structurée ${ }^{28}$ ? Un tel jeu révèle un recul et donc une analyse du procédé de moralisation en action dans les Bestiaires. Est ainsi mise en lumière la conscience que les choses ne sont pas toujours à prendre «au pied de la lettre " et qu'en conséquence l'établissement des correspondances allégoriques ressortit aussi à un procédé d'écriture.

C'est pourquoi l'exemple du Bestiaire me paraît révéler aussi l'exercice d'un art de la mémoire, une inscription de données compilées à usage d'une autre compilation. La correspondance animal/allégorie permet la détermination mémorielle d'un double savoir. D'un côté, à l'usage du prédicateur, l'image animale permet la fixation de l'exemplum réutilisable dans un sermon; de l'autre côté, la connaissance habituelle de l'Écriture permet la fixation d'une connaissance des choses de la nature, qui d'ailleurs pourra être utilisée en retour d'une façon exégétique, selon une autre interprétation que l'allégorie proposée par le Bestiaire. Thomas de Cantimpré, dont l'encyclopédie est centrée sur l'étude des animaux, indique bien dans son prologue qu'il entend écrire à l'usage des prédicateurs: les exemples de la nature, dit-il, pourront éveiller les âmes brutes jusqu'alors insensibles à la prédication habituelle. Certes ; mais, en même temps, ne peut-on comprendre que les choses de la nature forment aussi, pour le prédicateur, un ensemble de données exemplaires qui l'aideront à mieux structurer et à mieux mémoriser ses sermons?

21 Le parallèle entre le De natura rerum de Thomas et l'Ovide moralisé n'a rien de gratuit. En effet, Marc-René Jung a montré de façon très convaincante qu'une des lectures possibles de l'Ovide moralisé était celle, justement, du prédicateur. En analysant minutieusement le livre XI, le savant critique a établi que l'auteur de l'Ovide moralisé avait conçu sa moralisation comme un sermon et qu'ainsi «il parle en prédicateur " ${ }^{29}$. Reprenant l'enquête, Jean-Yves Tilliette s'est interrogé sur les sources des moralisations du texte, en direction, en particulier, des recueils de distinctiones. S'il aboutit à une impossibilité, dans l'état de la recherche, d'identifier des ressemblances formelles entre l'Ovide moralisé et de tels recueils, il émet cependant une hypothèse particulièrement suggestive. Les récits ovidiens, selon Tilliette, ne constituent pas une donnée première, indépassable, prétexte à l'accrochage d'un message édifiant. C'est un phénomène contraire qui se produit. C'est le «texte inscrit de toute éternité par Dieu dans le monde qui est premier. À charge pour l'interprète d'y adapter, mais après coup, les histoires, les fables $»^{30}$. Selon une telle approche, le texte apparaît comme une véritable construction, selon une démarche délibérée de l'auteur. Il est vrai que la lecture de l'Ovide moralisé révèle bien, surtout dans les premières parties du texte, un désir évident de faire coïncider la fable et l'histoire biblique ; en particulier la Genèse ovidienne et la Genèse vétérotestamentaire. Une telle démarche est de toute façon fort naturelle pour un auteur médiéval cherchant à multiplier les vérités coïncidentes : la cosmogonie ovidienne se retrouve, à l'état de trace au moins, dans les encyclopédies qui la mettent aussi en parallèle avec la Création, démarche exacerbée chez un Pierre 
Bersuire qui, dans son Reductorium morale, intègre un Ovidius moralizatus. Le parallèle avec les Bestiaires me parait ici encore pouvoir apporter quelques éclaircissements.

L'étude de Marc-René Jung, comme celle de Jean-Yves Tilliette montrent de toute évidence qu'il existe un «accrochage» de la moralisation à la fable ovidienne. D'un premier point de vue, l'allégorie construit le sermon qui s'illustre dans la fable. Par ailleurs, l'histoire religieuse préexiste, originelle et fondamentale, et la fable vient seulement l'illustrer, la renforcer. On peut alors s'interroger sur la nécessité d'un tel accrochage. Pourquoi, en elle-même, l'histoire sainte ne suffit-elle pas? Surtout si l'on songe, encore une fois, que les principaux lecteurs, dans cette perspective, sont des clercs, des gens d'Église.

Avant de répondre à une telle question, il me faut revenir sur un autre élément qui me paraît devoir intervenir dans l'analyse de l'Ovide moralisé. Ce texte en effet est, avant tout, un recueil de fables. Faisons retour à ce propos sur la réception médiévale des Métamorphoses. C'est selon deux axes que les Métamorphoses sont reçues par le Moyen Âge, qui correspondent d'ailleurs à ce qu'Ovide lui-même a inséré dans son texte. Les préoccupations savantes d'Ovide sont bien connues et il parait naturel qu'un public avide de découvertes venues des autorités antiques cherche chez Ovide un témoignage, caché sous la fable, des propriétés des choses de la nature ${ }^{31}$. Or l'auteur de l'Ovide moralisé s'inscrit, au XIVe siècle, dans la continuité de la démarche chère au monde des Écoles du XII ${ }^{e}$ siècle, visant à la glose, au commentaire des auteurs latins, qui se sont exprimés sous le voile de la fable. C'est l'époque de la recherche ad integumentum, à travers laquelle les savants du XII ${ }^{\mathrm{e}}$ siècle, à l'image des pratiques chartraines, s'efforcent de comprendre une nature (livresque) à laquelle ils s'éveillent à travers ce que les sages anciens ont pu mettre en mémoire pour leurs successeurs. Si l'Ovide moralisé apparaît comme une somme mythologique, l'œuvre peut être également lue, en dehors de la moralisation, comme contenant un certain nombre d'informations scientifiques, qui se disent à deux niveaux: le premier consiste en une simple lecture, au premier degré ; l'information, tout particulièrement dans le domaine des sciences du ciel, se livre à la lecture du texte. Le second, qui rejoint l'integumentum des Chartrains (l'«integument» de Jean de Meun) ne saurait se donner qu'à travers une glose «physique» que l'auteur fournira ou ne fournira pas. Ambiguïté d'un texte qui donc ne peut être classé comme une encyclopédie à part entière, mais qui, on le voit, s'en approche par sa dimension de «vulgarisation scientifique». Quoi qu'il en soit, le public des clercs est particulièrement concerné par ce type d'information.

L'auteur de l'Ovide moralisé indique lui-même sa dette envers cette conception de la fabula héritée du XII ${ }^{\mathrm{e}}$ siècle. Les fables «semblent mençoignables/Mais n'i a riens qui ne soit voir/ Qui le sens en porroit savoir,/La veritez seroit aperte/Qui souz la fable gist couverte » (vv. 42-46). Il insiste d'ailleurs à nouveau sur cet aspect, à la fin du texte :

Voirs est, qui Ovide prendroit

A la letre et n'i entendroit

Autre sen, autre entendement

Que tel com l'auctors grossement

I met en racontant la fable,

Tout seroit chose mençognable (XV, vv. 2525-31)

La question se pose de savoir de quelle vérité il s'agit; comme le long début cosmogonique, ainsi que les nombreuses digressions sur le ciel physique l'attestent, cette vérité est double : elle est celle de la nature des choses d'une part et de l'allégorie 
de ces choses d'autre part. La moralisation ouvre le chemin à ces deux types de vérité, grâce à l'énoncé de la fable. Dans l'Ovide moralisé, la fable synthétise et la moralisation analyse. La fable peut alors apparaître comme le signe de la leçon scientifique et/ou mythologique.

Mais si l'auteur traduit Ovide, ce n'est pas non plus simplement et exclusivement pour transformer les Métamorphoses en un accessus à l'astronomie ou en un recueil de sermons. C'est aussi parce que le texte ovidien présente un intérêt, disons historique, en lui-même. Comme on l'a vu, l'Ovide moralisé en prose porte témoignage de l'intérêt particulier porté aux fables ovidiennes. Dans ce dernier texte, on peut inverser les paramètres qui viennent d'être énoncés en considérant que l'allégorie peut fonctionner comme le signe de la fable.

On voit donc pourquoi la parole sacrée, dans ce type de littérature, ne «se suffit pas à elle-même ». La fable ovidienne, comme l'énoncé d'une liste animale dans les Bestiaires, possède plusieurs fonctions : 1) une fonction didactique : illustrer pour édifier ; 2) une fonction "encyclopédique »: raccorder des savoirs positifs à la parole biblique ; 3) une fonction de reconnaissance, à la fois générique et sociale : la récurrence d'un certain nombre d'invariants, la présence d'un processus de décryptage (l'allégorie) participent de l'identification, ou du rapprochement d'un genre donné (encyclopédique, didactique et moral), tout en contribuant à l'affirmation de l'autorité de l'auteur (celui de l'Ovide moralisé ne rappelle-t-il pas qu'il a réussi là où d'autres ont renoncé ?); cette autorité rejaillit sur le lecteur lui-même, qui acquiert ou approfondit la connaissance de ce qui est sous le voile de la fabula et qui, en même temps, reconnaît son appartenance au groupe de ceux qui partagent cette connaissance ; 4) une fonction de mémoire : le texte est un lieu de mémoire, recueil de fables fixant un certain nombre de concepts chrétiens utiles à la prédication ou à l'interprétation des Écritures, un ensemble de données savantes, une collection d'« hystoires».

Prologues

Toujours dans le cadre d'une étude comparative, en rapport avec la "dominante» encyclopédique, il convient de se pencher sur le prologue de l'Ovide moralisé, version versifiée et version en prose. En effet, comme j'ai tenté de le montrer ailleurs, le genre encyclopédique se définit aussi par un certain nombre de données permanentes apparaissant dans les prologues, formant un réseau d'«invariance prologuiale $»^{32}$. Ces invariants peuvent être classés en quatre ensembles principaux :

- captatio benevolentiae et soulignement du rôle du commanditaire, mise en retrait du compilateur

30 - affirmation de l'utilité de l'encyclopédie, généralement dans l'intention d'une lecture des Écritures

31 - renvoi aux autorités

32 - identification de l'ouvrage selon deux critères essentiels: la brièveté et le rassemblement de données compilées.

33 La captatio benevolentiae n'a rien de bien particulier en soi, mais, dans le contexte des encyclopédies, elle répond surtout à l'affirmation de l'opération de compilation, dont l'auteur est conscient. Cette captatio est aussi à relier, surtout dans le cadre des traductions du XIV siècle, à la valorisation du commanditaire. Dans la version en vers, l'auteur se livre à une traditionnelle déclaration d'humilité : 
Mes ains, pour ce que je me sens

De foible engin, de foible sens,

Proi tous ceulz qui liront cest livre,

Que, se je mespreng a escrire

Ou a dire que je ne doie,

Corrigent moi. Bien le vaudroie,

Et je suis prest, se Dieu m'ament,

De croire leur corrigement

Si com sainte yglise vouldra,

Que croire doi ce qu'el croira.

Qui autrement m'en reprendroit,

Je diroie qu'il mesprendroit (vv. 59-70)

Remarquons cependant que cette déclaration ne se limite pas au seul constat du «foible engin» de l'auteur. Ce dernier en effet situe son écriture dans une dimension évidemment didactique et laisse au lecteur le soin de juger de la qualité de son propos, en terme d'orthodoxie : il espère ne pas se méprendre sur ce qu'il va dire, c'est-à-dire qu'il entend ne pas se tromper sur la leçon. Et le juge n'est finalement pas le lecteur anonyme, mais "sainte yglise", soit avant tout la communauté des clercs. Ce topos d'humilité revêt une forme particulière qui ramène à l'écriture encyclopédique. Les auteurs en effet, tels Barthélemi l'Anglais, prétendent régulièrement mettre peu du leur et se placent sous l'autorité des saints et des philosophes. Le lecteur pourra comparer les dires de l'encyclopédiste avec les textes sources et, éventuellement corriger et/ou aller plus loin. Dans la version en prose, on retrouve, encore plus marquées, les caractéristiques topiques des prologues encyclopédiques et des prologues de traduction ${ }^{33}$, dans lequel le commanditaire, ici le roi René, est largement tenu pour responsable de la qualité du texte : «si lui supplie tres humblement qu'il luy plaise de sa benignité prendre en gré mon petit stille et rude ouvraige et en supporter et pardonner les fautes qui par mon ignorance y pourroient estre trouvées $»^{34}$.

L'auteur de l'Ovide moralisé déclare également, en entrée de prologue, faire œuvre utile et ce, à double titre : il va enseigner et il va expliciter les fables des poètes, traduisant le «latin en romans » (v. 16). Il se prétend guidé par la volonté divine, reprenant un topos encyclopédique tel qu'on le trouve, entre autres, chez Alexandre Neckam; et pour cela, il va faire ce à quoi d'autres ont échoué. Tout le début du prologue de l'Ovide moralisé en vers est orienté par cette déclaration de didactisme. Enfin, on trouve la déclaration de vérité (v. 45) et de brièveté :

Mes les mutacions des fables,

Qui sont bones et profitables,

Se Dieus le m'otroie, esclorrai

Au plus briement que je porrai,

Pour plus plaire a ceulz qui l'orront,

Et maint profiter y porront. (vv. 53-58)

Nous n'avons pas ici, comme dans le genre encyclopédique à proprement parler, une formulation de "somme brève" (summa brevis), mais cette intention de faire bref, intégrée dans un prologue qui reprend d'autres éléments topiques de l'invariance encyclopédique, fait écho. Si l'on ne peut raisonnablement parler d'invariance prologuiale encyclopédique pour l'Ovide moralisé, il me semble cependant qu'il est possible d'enregistrer un ensemble cohérent et convergeant, rassemblant, plus diffusément, plus parcellaire, un faisceau d'éléments de cette invariance. 

l'histoire sainte renforcée, soulignée par la coïncidence de la fable ovidienne, mémoire mythologique ancrée dans une interprétation allégorique religieuse, lieu de fabulae nécessitant un décryptage secundum integumentum, l'ovide moralisé apparait, en dépit d'une mauvaise réputation qui l'a longtemps déprécié, comme un texte particulièrement riche s'évasant en de multiples directions. Un des points de rencontre de ces différentes orientations me semble résider dans la construction, peut-être délibérée, en tout cas manifeste pour certains lecteurs, d'un lieu de mémoire à caractère encyclopédique, où un savoir se cristallise et s'organise dans un réseau de correspondances.

Ce savoir s'organise assez nettement, à quelques incohérences prêt - en particulier lorsque l'auteur se laisse aller à certaines critiques, comme celle des prélats qui par trop s'enrichissent. Les moralisations forment chacune une unité, faisant suite à l'énoncé de la fable, unité qui peut fonctionner de manière indépendante. L'auteur d'ailleurs prend bien soin de marquer ces unités par des formules récurrentes, dans lesquelles le narrateur intervient explicitement, formules du type «autre sentence $i$ puet avoir », "par...puet entendre », « or vou dirai... ». Ce marquage est encore plus net dans la version en prose, grâce à la rubrication du manuscrit. Ces formules me paraissent souligner la construction de l'ouvrage qui apparaît bien, en dépit de sa longueur, comme constitué d'unités séparées pouvant être lues avec une certaine discontinuité, mais sans pour autant que le texte, à la façon des encyclopédies du temps, soit consultable de façon parcellaire et non continue. On peut alors chercher dans l'Ovide moralisé, comme les poètes l'ont généralement fait, des informations mythologiques. On peut aussi y choisir des exempla, comme de véritables sermons. On peut y trouver encore des informations sur le monde et la nature et, de ce point de vue, l'ovide moralisé participe à sa façon à la stratification des textes encyclopédiques de la fin du Moyen Âge. Aux questions de classification de l'ovide moralisé, proposant des catégories telles que l'encyclopédie sur la nature, l'encyclopédie moralisée, l'encyclopédie religieuse, on ne pourra que répondre par la négative. Cependant, il existe, j'espère l'avoir montré, des «rapports de connexité » avec la tradition encyclopédique, tels ceux que l'on peut définir pour les Bestiaires ou les Lapidaires. En qualifiant l'Ovide moralisé d'encyclopédie moralisée de fables mythologiques, si l'on ne définit pas à proprement parler de nouveau genre, on souligne ces relations de connexité. Il est important alors de noter ces relations, car, si une telle perspective n'apporte pas d'information particulière sur le texte en lui-même, elle précise des éléments importants de sa réception et permet de mieux situer la place essentielle que l'ovide moralisé occupe, comme source dynamique, dans le didactisme qui baigne l'écriture de la fin du Moyen Âge.

\section{NOTES}

1.Publications de l'Académie royale néerlandaise, 1915-1938. 
2.M. Possamaï, «L'Ovide moralisé, une somme du début du XIV ${ }^{\mathrm{e}}$ siècle ? », Sommes et cycles, dir. M.-E. Bély, J.-R. Valette, J.-Cl. Vallecalle, Cahiers de l'Institut catholique de Lyon, 30, Lyon, 2000, pp. 147-64.

3.B. Ribémont, «Der Ovide moralisé : ein locus memoriae? », à paraître dans les Actes du colloque Schatzkammern des Wissens, (dir. D. Rieger, H. Krasser), Rauischholzhausen, oct. 2001.

4.Sur la définition de ce genre moralisé, voir mon ouvrage, Les Origines des encyclopédies médiévales. D’Isidore de Séville aux Carolingiens, Paris, Champion, Bibliothèque du Moyen Âge, 2001, p. 226sq.

5.B. Ribémont, Littérature et encyclopédies du Moyen Âge, Orléans, Paradigme, 2002, «Le Bestiaire et l'encyclopédie ", pp. 75-85.

6.P. Zumthor, Essai de poétique médiévale, Paris, Seuil, 1972, p. 160.

7.E.R. Jauss, «Littérature médiévale et théorie des genres », Théorie des genres, ouvrage collectif, Points Seuil, 1986, pp. 37-76.

8.Éd. C. de Boer, North-Holland, Amsterdam, 1954.

9.Art. cit., p. 44.

10. Ibid.

11.Je ne reviens pas ici sur la définition du genre encyclopédique. Je renvoie à mes travaux sur cette question, en particulier, «L'établissement du genre encyclopédique au Moyen Âge », Littérales, 21, 1997, pp. 189-207 et Les Origines..., op. cit.

12.Voir l'introduction de l'édition de C. de Boer pour l'Ovide moralisé en vers, qui comporte une étude des emprunts de Machaut. Encore sur Machaut, R. Trachsler, « la Sibylle et le chaînon manquant ", Romania, CXVI, 1998, pp. 188-214; voir également J.K. Kellogg, "Transforming Ovid. The metamorphosis of female authority ", Christine de Pizan and the categories of difference (ed. M. Desmond), Univ. of Minnesota Press, Minneapolis, 1998, pp. 181-94.

13.Sur la transposition, voir B. Ribémont, Introduction à Le livre des propriétés des choses, une encyclopédie au XIV ${ }^{e}$ siècle, Paris, Stock, 1999 et Les Origines..., op. cit.

14.L'exemple le plus frappant étant celui de Froissart, recréant un certain nombre de mythes ovidiens dans ses Dits, surtout dans l'Espinette amoureuse et le Joli buisson de Jonece.

15.Voir, entre autres, J. Cerquiglini, «Un engin si soutil ». Guillaume de Machaut et l'écriture au XIV ${ }^{e}$ siècle, Paris, Champion, 1984.

16.Voir l'introduction de mon édition du Dit de la panthere, Paris, Champion, CFMA, 2000 et «Insertion savante et 'encyclopédisme littéraire'. La lettre et la science dans l'écriture médiévale : l'exemple du roman antique ", à paraître dans Actes du colloque Réécriture littéraire des discours scientifiques, (dir. Ch. Foucrier, D. Mortier), Rouen, déc. 2001

17.B. Ribémont, «Christine de Pizan et l'encyclopédisme scientifique », The City of Scholars. New Approaches to Christine de Pizan, (dir. M. Zimmermann, D. de Rentiis), Berlin, 1994, pp. 174-85 ; «Christine de Pizan entre espace scientifique et espace imaginé (Le Livre du chemin de long estude) », Une femme de Lettres au Moyen Age. Études autour de Christine de Pizan, (dir. L. Dulac, B. Ribémont), Orléans, Paradigme, 1995, pp. 245-64. 18.M.-R. Jung a édité une large partie de ces gloses, «L'Ovide moralisé glosé », Literatur : Geschischte und Verstehen. Festschrift für Ulrich Mölk zum 60. Geburtstag, (Hrsg H. Hudde und U. Schöning), C. Winter, Heidelberg, 1997, pp. 81-93. 
19.Voir B. Van den Abeele, «Bestiaires encyclopédiques moralisés. Quelques succédanés de Thomas de Cantimpré et de Barthélemy l'Anglais ", Reinardus, 1994, pp. 209-28 ; H. Meyer, Die Enzyklopädie des Bartholomaüs Anglicus. Untersuchungen zur Überlieferungs-und Rezeptiongeschichte von De Proprietatibus rerum, W. Fink Vlg., München, 2000.

20.Éd. C. de Boer, North-Holland, Amsterdam, 1954.

21.Le texte est en effet parsemé de remarques concernant l'intervention du duc d'Anjou poussant l'auteur à abréger (VII, 5 ; IX, 2 ; XIV, 1, etc.).

22.Voir plus loin dans le texte.

23.Problématique que l'on retrouve au sein même de la tradition encyclopédique, avec les deux versions de l'Image du monde de Gossuin de Metz. Voir mon article, «Vers et prose dans l'écriture à caractère scientifique au Moyen Âge ", Congrès international de linguistique et de philologie romanes, Zürich, 6-11 avril 1992, publié dans les Actes, 1993.

24.Voir mon article, «Le monde animal dans l'Ymago mundi de Pierre d'Ailly ", Fifteen Century Studies, 19, 1992, pp. 229-42.

25.Voir, entre autres, F. Berier, «La traduction en français », GRLMA, VIII, 1, 1988, pp. 219-65 ; S. Lusignan, Parler vulgairement. Les intellectuels et la langue française aux XIII et XIV ${ }^{e}$ siècles, $2^{\mathrm{e}}$ éd., Paris, Vrin, Montréal, PUM, 1987.

26.Voir G. Ouy, Gerson bilingue, Paris, Champion, 1999.

27.B. Ribémont, Les origines..., op. cit ; La Renaissance du XII siècle et l'encyclopédisme, Paris, Champion, 2002.

28.Voir mon article, «Bestiaire d'amour et zoologie encyclopédique : le cas des abeilles (Bestiaire d'amour rimé anonyme et Bestiaire d'Amour de Richard de Fournival) ", Revue des Langues Romanes, 48, 2, 1994, pp. 341-68.

29.M.-R. Jung, "Aspects de l'Ovide moralisé », Ovidius redivivus. Von Ovid zu Dante, ed. M. Picone, B. Zimmermann, Stuugart, 1994, pp. 149-72.

30.J.-Y. Tilliette, «L'écriture et sa métamorphose. remarques sur l'Ovide moralisé ", Ensi firent li ancessor. Mélanges de philologie médiévale offerts à Marc-René Jung, (éd. L. Rossi), Delle'Orso, Alessandrina, 1996, pp. 543-58 ; cit. p. 555.

31.Bien que datant un peu, l'ouvrage de S. Viarre, La survie d'Ovide dans la littérature scientifique des XII ${ }^{e}$ et XIII ${ }^{e}$ siècles, Poitiers, 1966, reste un outil de travail fondamental. 32.Voir mon article, «Encyclopédie et traduction : le double prologue du Livre des proprietés des choses ", à paraître dans Seuils de l'œuvre (dir. E. Baumgartner, L. Harf), Presses de la Sorbonne nouvelle.

33. Voir Ibid.

34.Éd. cit, p. 43. 